\title{
Monolithic Solder-on Nanoporous Si-Cu Contacts for Stretchable Silicone Composite Sensors
}

Michael Kasimatis ${ }^{1,}{ }^{*}$, Estefania Nunez-Bajo ${ }^{1}$, Max Grell ${ }^{1}$, Yasin Cotur ${ }^{1}$, Giandrin Barandun ${ }^{1}, J_{\text {- }}$ Seon $\mathrm{Kim}^{2}$, Firat Güder ${ }^{l, *}$

${ }^{1}$ Department of Bioengineering, Imperial College London, London SW7 2AZ, United Kingdom

${ }^{2}$ Department of Physics, Imperial College London, London SW7 2AZ, United Kingdom

*CORRESPONDING AUTHORS:

M. Kasimatis, e-mail: $\underline{\text { m.kasimatis16@imperial.ac.uk }}$

Dr. F. Güder, e-mail: guder@imperial.ac.uk 


\begin{abstract}
We report a method of creating solderable, mechanically robust, electrical contacts to interface (soft) silicone-based strain sensors with conventional (hard) solid-state electronics using a nanoporous $\mathrm{Si}-\mathrm{Cu}$ composite. The $\mathrm{Si}$-based solder-on electrical contact consists of a copper-plated nanoporous Si top surface formed through metal-assisted chemical etching and electroplating, and a smooth Si bottom surface which can be covalently bonded onto siliconebased strain sensors through plasma bonding. We investigated the mechanical and electrical properties of the contacts proposed under relevant ranges of mechanical stress for applications in physiological monitoring and rehabilitation. We also produced a series of proof-of-concept devices, including a wearable respiration monitor, leg band for exercise monitoring and Squeezeball for monitoring rehabilitation of patients with hand injuries or neurological disorders, to demonstrate the mechanical robustness and versatility of the technology developed, in real-world applications.
\end{abstract}

Keywords: soft sensors and electronics, stretchable electronics and sensors, flexible devices, wearable sensors, monolithic integration 


\section{Introduction}

Stretchable electronics have produced a large number of mechanical, chemical and biological sensing elements, piezoelectric generators, and even photovoltaics since their conception in the late '90s. ${ }^{1-9}$ Stretchable devices can conform to curved surfaces (such as the human body) and instantly adapt to dynamic changes in the geometry of the surface they are attached to, hence they are exceptionally suitable as wearable devices for physiological monitoring and rehabilitation. ${ }^{10-12}$ Stretchable strain sensors constructed using electrically conductive and elastomeric polymer composites (CPC) can reversibly transduce mechanical deformations into an electrical signal through changes in their resistance or capacitance. Silicone-based stretchable CPC strain sensors are particularly easy to fabricate (in a standard laboratory environment - i.e. without the need for advanced facilities such as a cleanroom) and consist of conductive particle fillers dispersed in a silicone polymer matrix to measure applied strain. The sensors can be molded into the shapes desired using 3D-printed molds or printed directly at room temperature via screen or stencil printing. ${ }^{13,14}$ Among others, carbon black-filled polydimethylsiloxane (CB-PDMS) CPCs are widely used as strain sensors because of the longterm chemical and mechanical stability of silicones, tunable mechanical/electrical properties of these composites, and their low-cost.

Works reported to date have optimized the sensing properties and the manufacturing of stretchable CB-PDMS sensing elements, however, reliable interfacial adhesion of the stretchable sensing element with electronic components, such as wiring, integrated circuits, or other conventional devices, still remains a problem. ${ }^{15-17}$ Currently, the electrical connection between CB-PDMS sensors and external electronics primarily relies on brittle conductive adhesives, although some commercial products use large solid mechanical connections, such as clamps and 
clasps, that are not suitable for miniaturized devices (Smart Garments by Stretchsense ${ }^{\mathrm{TM}}$, Zephyr ${ }^{\mathrm{TM}}$ Biomodule ${ }^{\mathrm{TM}}$ Device).$^{18}$ Silver-based conductive epoxy (AgEpoxy) is, by far, the most widely used adhesive to create electrical contacts between silicone-based CPC sensing elements and electrical read-out devices. ${ }^{19-26}$ Even though AgEpoxy is an excellent electrical conductor $\left(<10^{-4} \Omega \cdot \mathrm{cm}\right)$, it is susceptible to cracking and debonding from the silicone-based stretchable strain sensors and usually the first interface to fail under rates of strain higher than $10 \mathrm{~mm} / \mathrm{min}(2$ $\mathrm{mm} / \mathrm{s}$ is physiologically relevant). ${ }^{27}$ This is due to the brittle nature of the adhesive and poor wetting of silicones by AgEpoxy. Silicones bond weakly with most adhesives due to their lower surface energy (the surface energy of epoxy is $40-50 \mathrm{~mJ} / \mathrm{m}^{2}$, while silicones have a range between $19-25 \mathrm{~mJ} / \mathrm{m}^{2}$ ). Hence, a reliable method is needed for bonding electronic components with silicones. ${ }^{28}$

To overcome the limitations of AgEpoxy, several groups explored vacuum deposition of metallic structures on silicone elastomers and subsequent soldering of wiring and components to achieve bonding. ${ }^{29-31}$ Vacuum deposition of metals on silicones, however, requires complex processes that generally involve patterning through photolithography, deposition of additional adhesion enhancement layers and liquid-phase transfer of films. Despite the complex fabrication methodologies, such metallic contacts are still susceptible to delamination and failure, especially in applications (such as physiological monitoring) where the contacts may experience high levels of applied stress (>1 MPa) and high rates of strain $(>2 \mathrm{~mm} / \mathrm{s}){ }^{32,33}$

In this work, we propose a method of creating solderable, mechanically robust, electrical contacts to interface (soft) CB-PDMS strain sensors with conventional solid-state (hard) electronics using a nanoporous $\mathrm{Si}-\mathrm{Cu}$ composite. The Si-based solder-on electrical contact consists of a copper-plated nanoporous Si top surface formed through metal-assisted chemical 
etching (MACE) and electroplating, and a smooth Si bottom surface which can be covalently bonded on silicone-based CPCs through one-step plasma bonding. We investigated the mechanical and electrical properties of the contacts proposed under relevant ranges of mechanical stress for applications in physiological monitoring and rehabilitation.

\section{Results and Discussion}

Fabrication of CB-PDMS: A range of concentrations between 5 and 20\% w/w CB in PDMSbase were prepared through mechanical mixing, sonication and solvent evaporation (Figure 1A). The CB-PDMS base was mixed with the curing agent and a $0.1 \mathrm{~mm}$ conductive layer was deposited on a carrier PDMS layer through elastomer-on-elastomer printing using a stencil mask. Both materials were then co-cured in a conventional oven at $100^{\circ} \mathrm{C}$ for 1 hour (see Supporting Information - Figure S1 for photographs of the printed and co-cured devices). ${ }^{34}$ We fabricated piezoresistive-type strain sensors because they are easily implemented into electrical read-out systems. The fabrication process developed, however, is versatile and can be adapted to different geometries and sensing modalities, including piezocapacitive sensors, and multilayer structures. We characterized the electrical resistance of unstrained CB-PDMS using a commercially available (Uni-Trend Technology Co., Ltd UT171B) multimeter (current measurement with 5 V applied voltage) and flat alligator clips, with a CB concentration range between $5-20 \%(w / w)$ of PDMS (Figure S2). The electrical resistance of CB-PDMS decreased rapidly with increasing concentration of $\mathrm{CB}$ particles from $9.5 \pm 1.5 \mathrm{M} \Omega$ for $5 \%$ to $4.1 \pm 0.3 \mathrm{k} \Omega$ for $20 \% \mathrm{CB}$ due to formation of conductive percolative networks within the polymer matrix, reaching values around $200 \mathrm{k} \Omega$ for $12 \% \mathrm{CB} .^{35}$ 
A

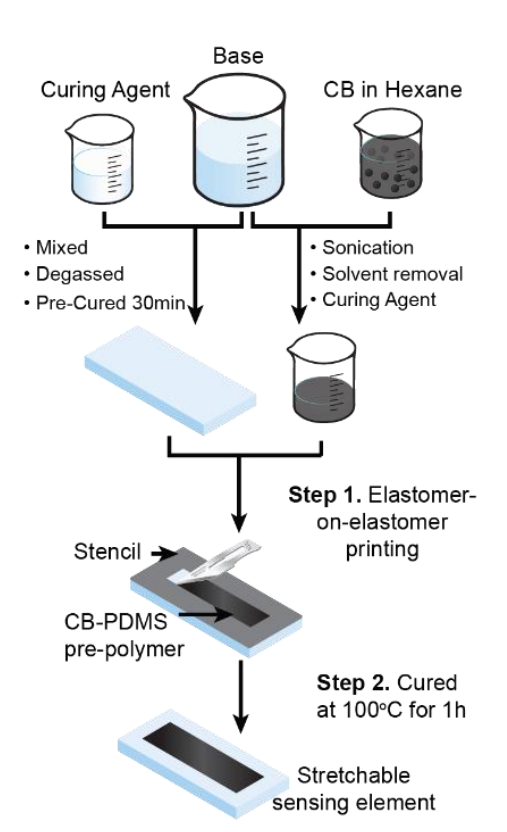

B

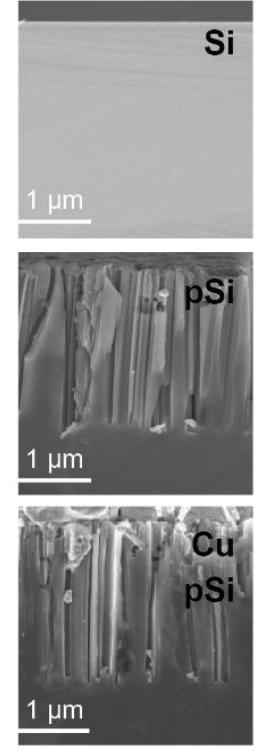

C

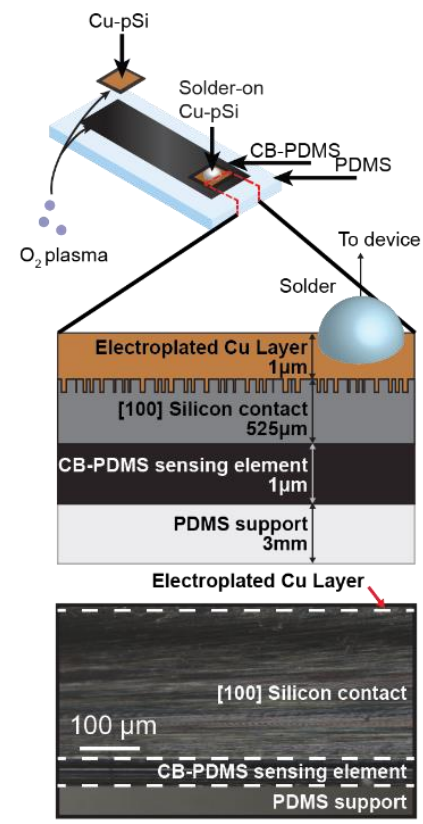

Figure 1: (A) Schematic representation of the fabrication of the stretchable silicone-based piezoresistive sensing element. (B) Cross-sectional SEM images taken during MACE and $\mathrm{Cu}$ electroplating on p-type Si (100) wafer. (C) Schematic illustration of (top) and cross-sectional optical micrograph (bottom) of $\mathrm{Cu}-\mathrm{pSi}$ contacts after plasma bonding on the CB-PDMS sensing element.

Si-based solder-on electrical contacts for silicone-based CPC sensors: The copper-plated nanoporous silicon $(\mathrm{Cu}-\mathrm{pSi})$ contacts were produced in a two-step process (Figure 1B): i) The top surface of a pristine p-type (100) silicon wafer was cleaned, electroless plated with Ag particles and placed in an etching solution (containing $\mathrm{H}_{2} \mathrm{O}_{2}$ and $\mathrm{HF}$ ) for 7 min to create a nanoporous Si surface through MACE. ${ }^{36,37}$ The surface resulting from etching consisted of 50$300 \mathrm{~nm}$ wide pores with a depth of $\sim 2.40 \mu \mathrm{m}$. ii) The nanoporous surface was electroplated in a $0.8 \mathrm{M} \mathrm{CuSO}_{4}$ aqueous bath $v s$. a Pt wire, to create a $\sim 1 \mu \mathrm{m} \mathrm{Cu}$ film on the nanoporous $\mathrm{Si}(\mathrm{Cu}-$ pSi) surface that can be soldered to external wiring and components. Elemental analysis by 
energy dispersive X-ray spectroscopy (EDX) and close-up electron micrographs show a clear separation between the $\mathrm{Si}$ and $\mathrm{Cu}$ surfaces for unetched $\mathrm{Si}$. For $\mathrm{pSi}$, however, the $\mathrm{Cu}$ layer extends inside the porous surface with decreasing amounts with increasing depth, likely due to limitations in mass transfer during electrodeposition (Figure S3). This limitation could be overcome through further optimization of the process parameters or using other electroplating regimens such as pulsed electrodeposition. ${ }^{38}$ The nanostructured surface plays the important role of improving the adhesion of the $\mathrm{Cu}$ layer without any additional adhesion enhancement layers on the Si surface, by increasing the surface area and morphological complexity to create interlocking structures. We performed tensile testing by soldering a $4 \mathrm{~mm}$ thick multicore $\mathrm{Cu}$ wire on the electroplated $\mathrm{Cu}$ layer on the surfaces of unetched $\mathrm{Si}$ and nanoporous Si with varying pore sizes. We did not observe a difference on adhesion strength of the $\mathrm{Cu}$ layer on $\mathrm{pSi}$ when we increased the pore size since in each experiment the $\mathrm{Si}$ substrate failed before the $\mathrm{Cu}$ film could detach from the surface. The unetched $\mathrm{Si}$ surface electroplated with $\mathrm{Cu}$, however, delaminated during handling, hence we were not able to perform tensile testing for these samples (please see Figure S4 for more information).

Electroplated wafers were cut into $5 \times 5 \mathrm{~mm}^{2}$ pieces by laser and the unmodified side of each piece (smooth, non-plated, non-porous side of the starting Si wafer) was plasma bonded to the CB-PDMS (Figure S5). Treating the surface of unmodified $\mathrm{Si}$ and CB-PDMS with $\mathrm{O}_{2}$ plasma for $35 \mathrm{~s}$ forms $\mathrm{SiO}_{\mathrm{x}}$ species on the surface and when in contact, the materials irreversibly bond together through a condensation reaction. ${ }^{39}$ The multilayered device, including the CBPDMS sensing element and Cu-pSi solder-on contact are shown in Figure 1C. Because plasma treatment modifies the mechanical properties of PDMS (increased brittleness - Table S1), a physical mask was used to limit areas of exposure to plasma, only at the sites of bonding. 
AgEpoxy samples were also plasma treated for equivalence as it is a common industrial method to improve bonding. ${ }^{40}$

(Electro)mechanical characterization of contact reliability under stress: We performed shear strength testing to compare the mechanical characteristics of $\mathrm{Cu}-\mathrm{pSi}$ and AgEpoxy electrical contacts on the interface of both with the silicone-based CPC (please note that we used AgEpoxy to attach a copper wire to the silicone-based CPC to pull the substrate and make electrical measurements). The samples were stretched from each end until the device-under-test (DUT) failed mechanically (Figure 2). First (Figure 2A), we investigated the effect of CB content (0$20 \%$ ) on the maximum stress to failure. CPC devices fabricated using the $\mathrm{Cu}-\mathrm{pSi}$ electrical contacts exhibited an increase in maximum stress $(n=5)$ to failure with increasing $\mathrm{CB}$ content in the composite while the maximum stress to failure for samples with AgEpoxy contacts ( $\mathrm{n}=5)$ did not change with increasing $\mathrm{CB}$ in the composite conductive layer. Strain stiffening in layered composites can be used in the future to form contact strain relief islands. ${ }^{41}$ This result indicates that each system has a different mode of failure (e.g., failure of the contact-substrate interface, failure of the CPC substrate itself, etc.). To investigate the mode of failure of the CPC the devices with $\mathrm{Cu}-\mathrm{pSi}$ and $\mathrm{AgEpoxy}$ contacts in more detail, we characterized the mechanical behavior of the devices under shear stress up to $40 \%$ strain (Figure 2B and Figure S6). We used silicone-based CPCs with $12 \%$ CB content for these experiments because it falls in the desired range of electrical resistance $\sim 200 \mathrm{k} \Omega$ - see Figure $\mathbf{S 2}$ (a current in the $\mu \mathrm{A}$ range is desired for further experiments using a $5 \mathrm{~V}$ Arduino). We have discovered that the samples with $\mathrm{Cu}-\mathrm{pSi}$ contacts produced a highly repeatable, linear strain-stress behavior up to approximately 0.95 MPa (or nearly 35\% strain) after which the DUT failed catastrophically with further increases in stress. On the contrary, the CPC devices with AgEpoxy contacts exhibited repeatable and linear 
stress-strain behavior only up to $0.37 \mathrm{MPa}$ (or 15\% strain). Further increases in strain caused devices with AgEpoxy contacts to slowly fail (i.e. no catastrophic failure in this case) as indicated by decreasing stress beyond $15 \%$ strain. Visual inspection of the samples before and after shear testing (Figure 2 B1, B2) revealed that, in the case of AgEpoxy contacts, the electrical contact - CPC interface failed slowly due to the brittleness of AgEpoxy and noncovalent, weak bonding between the adhesive and silicone substrate. In devices with $\mathrm{Cu}-\mathrm{pSi}$ contacts, the catastrophic mechanical failure was caused by fracturing of the silicone-based CPC and not the $\mathrm{Cu}-\mathrm{pSi}$ contact, suggesting that the bonding strength of $\mathrm{Cu}-\mathrm{pSi}$ contacts was higher than the yield strength of the silicone-based CPC. We further studied the mechanism of failure of the silicone-based substrate (CB-PDMS on PDMS carrier substrate) under tensile stress; regardless of the thickness of the PDMS carrier substrate, plasma-treated PDMS always fails around 0.5-0.7 MPa (Figure S7). We speculate that when subjected to plasma, PDMS becomes more susceptible to forming microscale cracks on its (somewhat hardened) surface. These cracks may subsequently propagate under applied tensile stress (normal to the direction of the crack) and cause failure (i.e. Mode I type crack propagation) ${ }^{42}$ This claim is further supported by the fact that increasing $\mathrm{CB}$ content in the $\mathrm{CB}-\mathrm{PDMS}$ also increased the maximum stress to failure (Figure 2A) since composite materials are more resistant to Mode I type crack propagation. ${ }^{43}$ Given that the substrates are flexible, for numerical analysis of the system, a superposition of Mode I and II loading should be considered. 
A

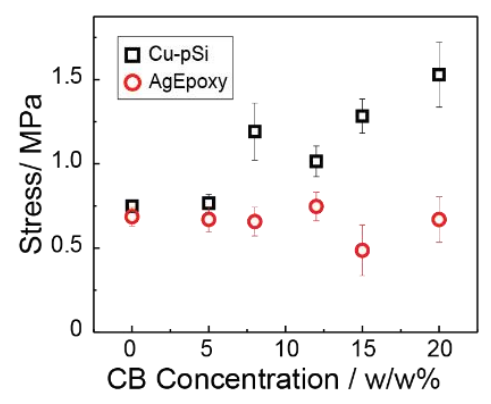

B

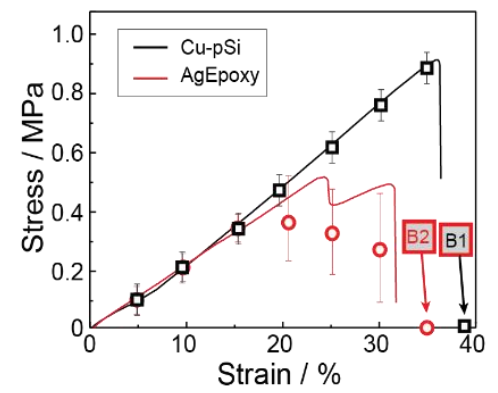

C

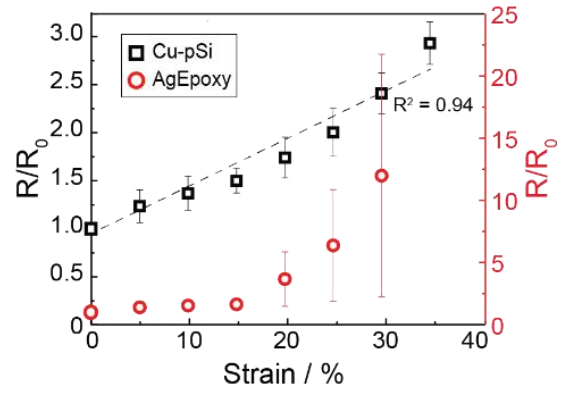

Sample $\stackrel{\text { Stress }}{\longrightarrow}$ Fracture
B1 Cu-pSi contact

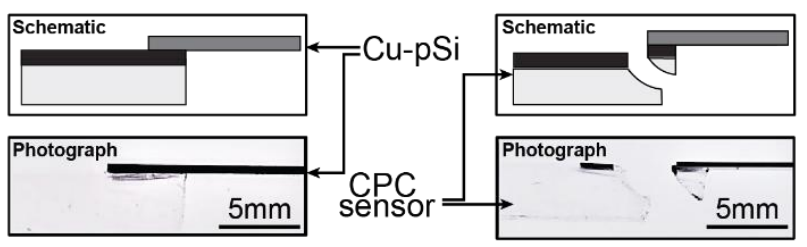

B2 AgEpoxy contact

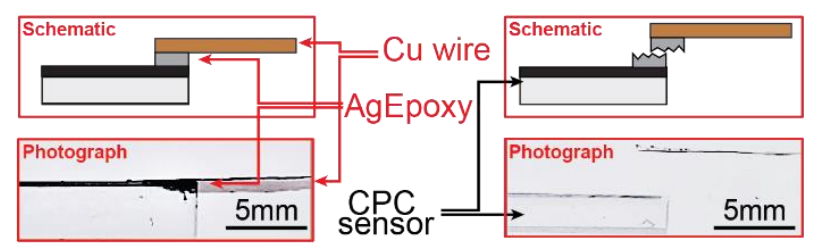

Figure 2: (A) Dependence of maximum stress to failure of Cu-pSi and AgEpoxy contacts in relation to CB filler concentrations ( $\mathrm{n}=5$ ) in the CB-PDMS composite; (B) Stress vs. strain for CB-PDMS composite with AgEpoxy and $\mathrm{Cu}-\mathrm{pSi}$ contacts during a single stretch to failure $(\mathrm{n}=7)$. (B1, B2) Modes of failure for CB-PDMS composites (12\% CB content) with AgEpoxy and $\mathrm{Cu}$ pSi contacts used in the experiments shown in (B); (C) Electromechanical characterization of 12\% CB-PDMS using different with Cu-pSi and AgEpoxy contacts (n=9).

Figure 2C illustrates the electrical resistivity-strain behavior of 12\% CB-PDMS devices connected to read-out electronics via copper wiring soldered on $\mathrm{Cu}-\mathrm{pSi}$ or adhered with AgEpoxy (see Figure S8). We measured the electromechanical characteristics during a single stretch to failure at a strain rate of $2 \mathrm{~mm} / \mathrm{s}$ (physiologically relevant rate for breathing). ${ }^{33}$ The resistance response of the CB-PDMS stretchable conductive layer (Response $=\mathrm{R} / \mathrm{R}_{0}$ where $\mathrm{R}$ is the measured resistance and $\mathrm{R}_{0}$ is the initial or baseline resistance) with $\mathrm{Cu}-\mathrm{pSi}$ contacts produced highly repeatable and linear response up to a maximum strain of 35\% (please note that 
beyond 35\% strain the devices fail catastrophically). Sensors with AgEpoxy, however, produced a highly-nonlinear response across the range of strains between 0-30\% due to gradual mechanical failure of the AgEpoxy electrical contact beyond 15\%. Hence, in all electromechanical tests performed, $\mathrm{Cu}$-pSi appeared to be more robust compared to AgEpoxy electrical contacts for silicone-based CPCs.

Characterization under cyclic stress: Since physiological monitoring (such as breathing) or rehabilitation exercises often involve repeated application of mechanical stress, we also characterized the electromechanical behavior of CB-PDMS strain sensors with $\mathrm{Cu}-\mathrm{pSi}$ and AgEpoxy contacts under cyclic stress. We subjected each sample to 20 cycles of repeated deformation at a strain rate of $2 \mathrm{~mm} / \mathrm{s}$ at each level of strain starting from $5 \%$ to account for stress relaxation in a method modified from Seyedin et al ${ }^{44}$ After every 20 cycles, the level of strain was increased by $5 \%$. The procedure was repeated until the sample (gradually or catastrophically) failed. Figure 3A shows a representative cyclic stress measurement (see Figure S9 for repeats for $\mathrm{n}=5$ ) of the sensors with $\mathrm{Cu}-\mathrm{pSi}$ and AgEpoxy contacts. Under cyclic stress, the devices with AgEpoxy contacts started failing gradually at strains higher than 10\% with complete failure beyond $15 \%$, barely withstanding $\sigma=0.20 \mathrm{MPa}$ (we captured gradual cracking and failure of the brittle AgEpoxy contacts also in a video - see supplementary Video V1). Devices with Cu-pSi contacts, however, would only fail at $30 \%$ strain $(\sigma=0.45 \mathrm{MPa})$ and remain fully functional under repeated strain until catastrophic failure of the substrate (after about 100 cycles at strains lower than 30\%). The electrical measurements under cyclic stress (Figure 3B) also followed a similar trend. At 15\% strain, the resistance slowly decreased and was eventually lost for the CPC devices with AgEpoxy contacts before reaching 20 cycles whereas the sensors produced with Cu-pSi contacts could reach 30\% strain before failing 
abruptly. The sensors with $\mathrm{Cu}$-pSi contacts, however, produced a non-linear electrical response with hysteresis especially when subjected to high levels of cyclic stress (>20\%) owing to the Mullins effect (i.e. softening due to previous loading) ${ }^{45,46}$ Since this mechanical effect is prevalent in all in-filled rubbers regardless of the type of contact, we expect that the devices with AgEpoxy contacts would also exhibit a similar behavior of hysteresis at higher strains if they could withstand such levels before failure.

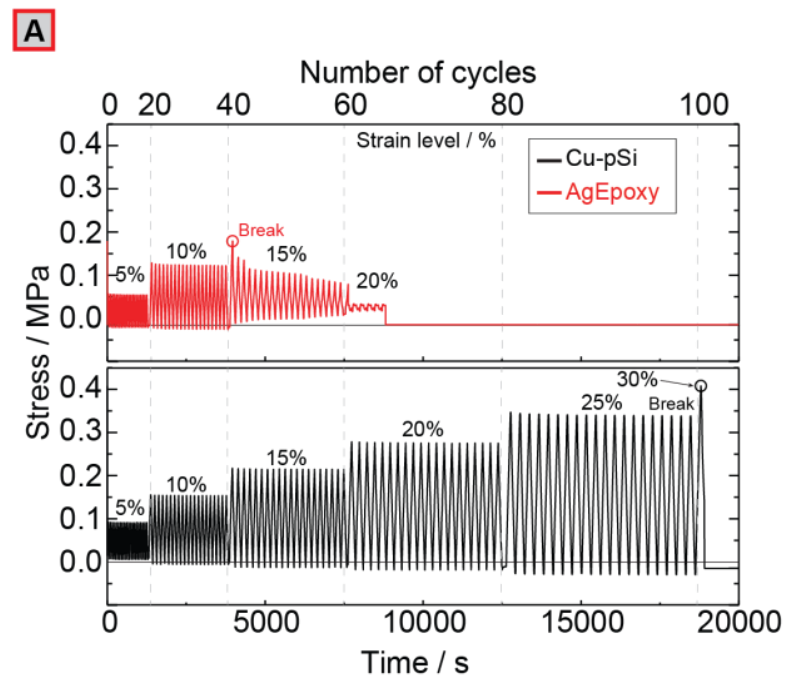

$B$

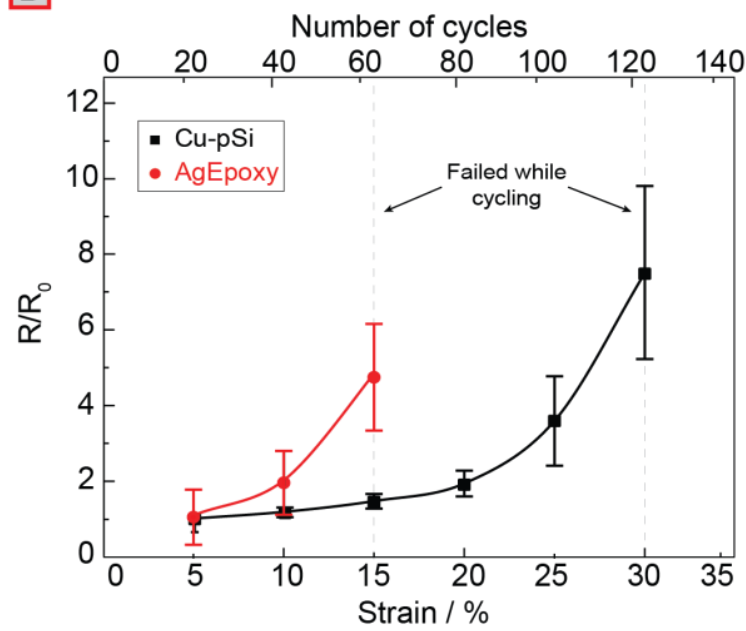

Figure 3: (A) CB-PDMS composite with AgEpoxy and Cu-pSi contacts subjected to cyclic strain; AgEpoxy (top) showing gradual failure beyond 15\% strain, and $\mathrm{Cu}-\mathrm{pSi}$ (bottom) showing 
abrupt failure beyond 30\% strain. (B) Electrical resistance after 20 cycles of strain and release at different strain levels $(n=5)$.

\section{Modelling of an equivalent electrical circuit:}

To understand the electrical behavior of the devices formed and to construct an equivalent circuit model, we performed 2-probe I-V measurements using CB-PDMS-based strain sensors with two $\mathrm{Cu}$-pSi contacts. The results of the I-V measurements are shown in Figure 4A. Because of the metal-semiconductor-metal nature of each electrical junction (i.e. $\mathrm{Cu}-\mathrm{Si}-\mathrm{C}$ ), the $\mathrm{I}-\mathrm{V}$ measurements $(\mathrm{n}=7)$ were highly non-linear (i.e. non-ohmic). In the range $-2 \mathrm{~V}$ to $2 \mathrm{~V}$, the devices exhibited a diode-like behavior due to the formation of Schottky barriers (because of the metalsemiconductor interface) with an exponential increase in electrical current for voltages beyond $0.5-1 \mathrm{~V} .{ }^{47} \mathrm{An}$ applied voltage with a magnitude higher than $1.0 \mathrm{~V}$ is therefore required for strainsensing with the $\mathrm{Cu}-\mathrm{Si}-\mathrm{C}$ junction. The $\mathrm{Cu}-\mathrm{Si}-\mathrm{C}$ contact structure leads to Schottky junctions at both $\mathrm{Si}$ heterointerfaces $(\mathrm{Cu}-\mathrm{Si}$ and $\mathrm{Si}-\mathrm{C})$, for both p-type and n-type $\mathrm{Si}$ (equilibrium diagram shown in Figure 4B). Fermi level pinning causes bending in the Si conduction and valence bands, which restricts the conductance at low potentials. The devices constructed using p-type $\mathrm{Si}$, however, were substantially more conductive than the devices created using n-type Si beyond the voltage of operation (i.e. $>1 \mathrm{~V}$ ). This is because the injection barrier height for the metal-Si interface for $\mathrm{p}$-type $\mathrm{Si}$ is lower than that of n-type $\mathrm{Si}$, leading to overall larger currents at the same potentials for devices with similar doping levels. ${ }^{48}$ The I-V graph for the junctions constructed using p-type Si indicates a more asymmetric barrier properties due to the differences in work functions $\left(\Phi_{\mathrm{M}}\right)$ of $\mathrm{Cu}$ and $\mathrm{C}$. For example, in the case of p-type $\mathrm{Si}$, because $\mathrm{Cu}$ has a work function $\Phi_{\mathrm{M}-\mathrm{Cu}}$ of $4.65 \mathrm{eV}$ and $\mathrm{C}, 4.81 \mathrm{eV}$ the barrier height for the $\mathrm{Si}-\mathrm{Cu}$ junction is larger. ${ }^{49}$ The height of the Schottky barrier can be reduced by using Si with higher doping levels, 
leading to higher charge carrier density, thereby reducing the width of the depletion layer. Using thin Si layers $(<100 \mu \mathrm{m})$ as contacts would also effectively reduce the total width of the depletion layer, as the depletion layers for each junction would begin to overlap. An equivalent circuit diagram is presented in Figure 4C representing the circuit where the CB-PDMS piezoresistive element is connected via the solder-on $\mathrm{Cu}-\mathrm{pSi}$ contacts to a power source. Since, regardless of the polarity of the potential applied, one of the junctions is always reverse-biased, the entire device can be modelled as a variable resistor with two Schottky diodes connected in reverse polarity. Please note that the model proposed is an over-simplification of the system, however, it represents the electrical behavior of the devices measured with sufficient accuracy.

A

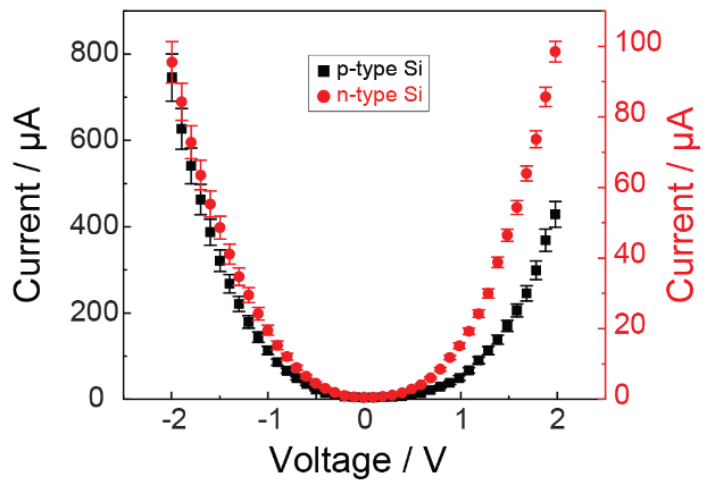

B

C

p-type

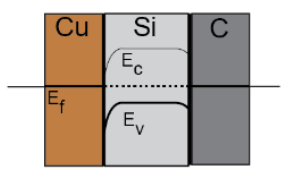

n-type
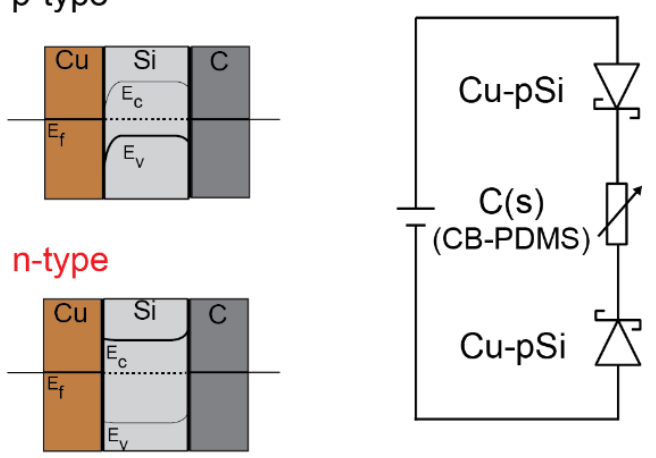
Figure 4: (A) I-V measurements for a CB-PDMS (12\% CB content) strain sensor with $\mathrm{Cu}-\mathrm{pSi}$ contacts created using lightly doped p-type and n-type Si (100) (n=7); (B) Equilibrium band diagrams for the $\mathrm{Cu}-\mathrm{Si}$-C interface. (C) Equivalent electrical circuit for the CB-PDMS strain sensor with two $\mathrm{Cu}-\mathrm{pSi}$ contacts.

Demo devices and applications: We have fabricated a series of proof-of-concept devices to demonstrate the mechanical robustness of the technology we developed in real-world applications (Figure 5). In the first example, we printed a CB-PDMS (12\% CB) conductive layer with $\mathrm{Cu}$-pSi contacts on an unmodified (non-conductive) silicone-based chest strap (Smooth-On Inc., Ecoflex ${ }^{\circledR}$ 00-30 used as carrier substrate) to measure breathing rate (supplementary Video V2) to demonstrate the applicability of our technology in wearable physiological monitoring. ${ }^{8}$ Figure 5A shows the change in electrical resistance during inhalation and exhalation due the expansion and contraction of the chest. During inhalation, as the chest expands, the strain sensor stretches, which leads to an increasing in the resistance of the CB-PDMS sensing element. As soon as the exhalation starts, the volume of the chest decreases leading to a decrease in the electrical resistance. Every peak in the signal corresponds to a cycle of respiratory activity. We were able to monitor breathing at rest (normal breathing), during fast and paused breathing, as well as heavy panting using the device we developed. Other devices, with a similar form-factor to the chest strap, can also be produced for various applications in exercise monitoring and rehabilitation such as a leg band (Figure 5B and Video V3). In another example we fabricated a silicone-based, ball-shaped device (i.e. Squeeze-ball) with embedded CB-PDMS (12\% CB) and $\mathrm{Cu}-\mathrm{pSi}$ contacts for monitoring rehabilitation of patients with hand injuries or neurological disorders such as stroke (Figure 5C). Squeezing the ball compresses the sensing element embedded inside which in turn changes the resistance relative to the force applied 
(supplementary Video V4). Although we did not fully quantify, the sensors were sensitive to both light and tight grip forces and could function reliably for repeated application of force through squeezing of the ball.

A Strain Sensing - Wearable Breathing harness

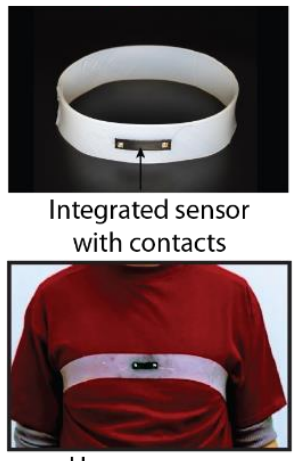

Harness worn around the chest

B Strain Sensing - Leg Band

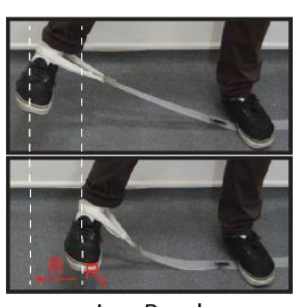

Leg Band Extension Measurement

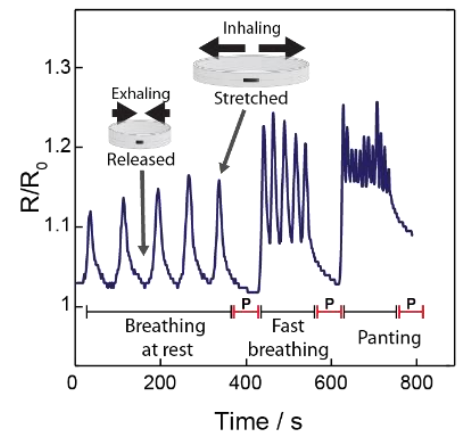

Time / s

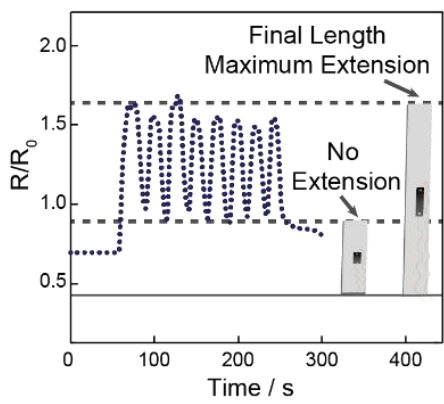

C Compression Sensing - Rehabilitation Squeeze-ball

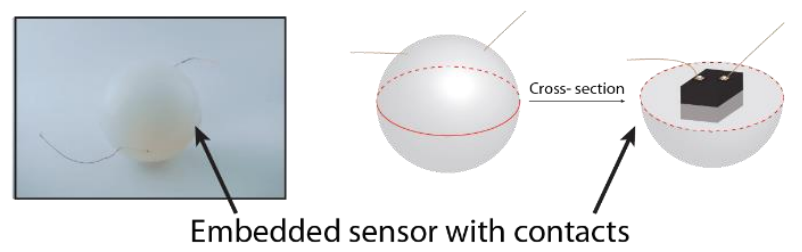

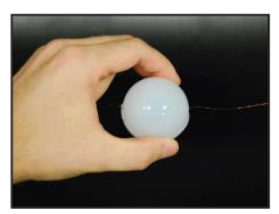

Ball in hand

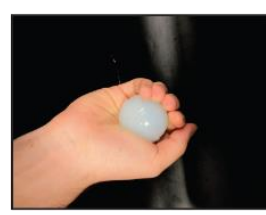

Soft Grip

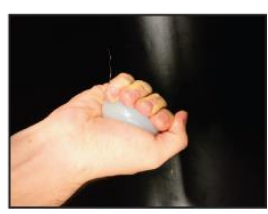

Tight Grip
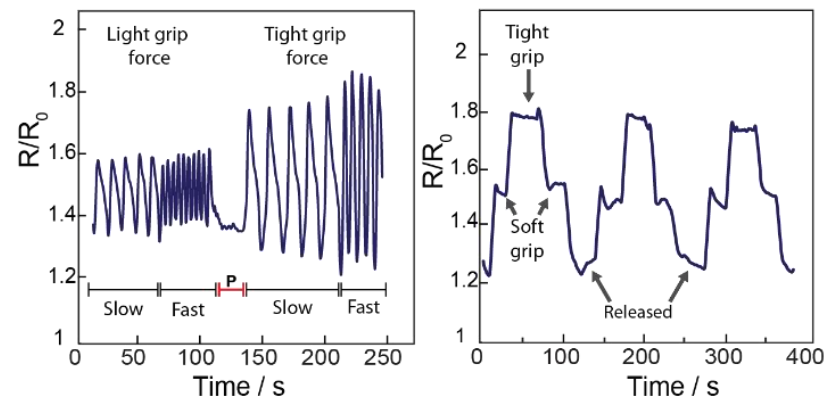

Figure 5: (A) Picture of completed stretchable harness and human subject wearing the device (i.e. CB-PDMS sensor with $\mathrm{Cu}-\mathrm{pSi}$ contacts); Physiological data collected from chest during inhalation (stretched) and exhalation (released) recorded with the harness in real-time; (B) Picture of a leg band containing the CB-PDMS sensor with $\mathrm{Cu}-\mathrm{pSi}$ contacts during a leg extension exercise. The electrical signals indicate the level of extension; (C) Picture and graphical illustration of the cross-section of the rehabilitation ball and a subject holding the ball for size comparison; Resistance data (equivalent to force) collected using the rehabilitation ball during exercise at light and tight gripping force at different exercise rates; Testing the sensitivity 
of the device by applying different grip forces (Soft grip $\rightarrow$ lower force, Tight grip $\rightarrow$ higher force). $\mathrm{P}=$ Paused.

\section{Conclusions}

In conclusion, we have presented a new technology for creating mechanically robust, covalently-bonded, Si-based electrical contacts for silicone-based stretchable CPC sensors and electrical devices. Unlike the existing solutions, the technology proposed is reliable, low-cost (cost of materials for two $5 \times 5 \mathrm{~mm}^{2} \mathrm{Cu}-\mathrm{pSi}$ contacts is $\$ 3 \times 10^{-3}$ ) and simple, consisting of only three steps for the fabrication and attachment of the contacts in a standard laboratory environment - i.e. formation of nanoporous $\mathrm{Si}$, electroplating with $\mathrm{Cu}$ and plasma-bonding on silicone-based conductive composites. The $\mathrm{Cu}-\mathrm{pSi}$ contact technology is compatible with largevolume manufacturing through the use of large Si wafers and has the potential to be the enabler of new classes of miniaturized silicone-based commercial stretchable devices with monolithically-integrated electrical contacts.

One of the most important features of the Cu-pSi technology is that the contacts do not fail gradually (regardless of whether the devices created are subjected to repeated, increasing or sustained mechanical stress) but instead fail catastrophically. This means that the devices produced using the $\mathrm{Cu}-\mathrm{pSi}$ technology will either produce a correct signal or do not produce a signal at all; this is especially important for applications in healthcare - e.g., disease diagnostics, rehabilitation monitoring - where wrong signals may lead to a different course of medical intervention.

The Cu-pSi contact technology, however, has the following three disadvantages: i) For the moment, $\mathrm{Cu}$-pSi can only be covalently bonded to silicone-based CPCs, therefore more 
research is needed for using this solution for other elastomeric materials. ii) Formation of nanoporous Si using metal-assisted chemical etching requires HF, a highly toxic chemical. This process, however, can be performed with HF solutions with a lower concentration, in-line with household cleaning products. ${ }^{50}$ Surface roughening for Si may also be performed using lesshazardous compounds such as $\mathrm{KOH}$ or potentially even mechanically, eliminating the need for HF. iii) Si substrates we used in this study were not stretchable nor flexible, but using stressdiffusion geometries and thin layers, Si can be rendered highly flexible and to some extent also stretchable.

In the future, the method reported will enable fabrication of highly reliable, batterypowered, portable, wireless, low-cost and integrated sensing systems using silicone-based stretchable sensors. Using a mobile device, such as a smartphone, the data generated by the sensors can be easily processed and stored on the cloud with capabilities for remote-access, important for applications in connected/digital healthcare. We believe, the Squeeze-ball (Figure 5C) in particular, can find immediate applications in the rehabilitation of patients with neurological diseases. Of course, this is just one application and the method proposed can find other uses such as performance monitoring in athletics, patient monitoring at home or hospitals.

\section{Experimental Methods}

Preparation of CB-PDMS composite. A suspension of powdered CB (50-nm particle size, Vulcan XC 72 Cabot Corporation), was mixed with PDMS (Sylgard 184, Corning Inc.) in a five step process modified from previously reported works ${ }^{51-53}$ : i) CB was suspended in hexane in a 1:10 (w/w) ratio and sonicated at $220 \mathrm{~W}$ for $10 \mathrm{~min}$. ii) Base elastomer was also dissolved in hexane in a 1:2(w/w) ratio in a different container. iii) The $\mathrm{CB}$ suspension was mixed with the 
base-solution (from step ii) in different amounts to produce 5, 8, 10, 12, 15, and 20\% (w/w) CBPDMS precursor. iv) Dispersions were dried in air overnight to remove the solvent - i.e. hexane.

v) The cross-linker was added to form the CB-PDMS composite polymer.

Preparation of PDMS carrier substrate. A PDMS substrate was prepared by mixing degassed base elastomer and cross-linker in a 10:1 ratio (w/w). The mixture was casted on a mold treated with Ambersil PTFE mold-release and pre-cured at $100{ }^{\circ} \mathrm{C}$ for $30 \mathrm{~min}$. As a result, a PDMS carrier substrate with a thickness of $3 / 6 / 9 \mathrm{~mm}$ was obtained and cut into strips of $10 \times 30 \mathrm{~mm}^{2}$ using a scalpel.

Assembly of strain sensitive CB-PDMS composite and PDMS carrier substrate. A plastic stencil with a rectangular pattern of $50 \mathrm{~mm} \times 5 \mathrm{~mm}$ was cut using a $\mathrm{CO}_{2}$ laser cutter (Universal Laser Systems, Inc. Model: V460, 60W). The stencil produced was used to print a 0.1-mm-thick CB-PDMS-based piezoelectric strain sensitive layer (sensing element) on the PDMS carrier substrate. The device with the printed CB-PDMS pattern was subsequently co-cured at $100{ }^{\circ} \mathrm{C}$ for $1 \mathrm{~h}$ to form the final structure.

Preparation of Cu-pSi contacts. Silicon wafers (p-type and n-type, Siegert Wafer, $525 \pm 25 \mu \mathrm{m}$ thickness, $0-100 \Omega \cdot \mathrm{cm}$, lightly doped) were cleaned with acetone, rinsed with distilled water then immersed in a 3:1 piranha solution $\left(95 \% \mathrm{H}_{2} \mathrm{SO}_{4}: 30 \% \mathrm{H}_{2} \mathrm{O}_{2} \mathrm{v} / \mathrm{v}\right)$ at $80{ }^{\circ} \mathrm{C}$ to remove organic residues. The wafers were dried in air using a blow dryer; one of the sides was protected with polyimide tape and transferred into a bath containing a solution of $2.9 \mathrm{mM} \mathrm{AgNO}$ for electroless plating of Si with Ag for 2 min to deposit metal particles on the surface. The wafers were rinsed and dried again and immersed in an etching solution of $2.72 \mathrm{M} \mathrm{HF}$ with $0.20 \mathrm{M} \mathrm{H}_{2} \mathrm{O}_{2}$ for 7 min to form nanoporous silicon (pSi) by Metal-Assisted Chemical Etching (MACE). ${ }^{36,54,55}$ Following etching, the wafers were treated with $10 \% \mathrm{HNO}_{3}$ to remove all $\mathrm{Ag}$ from the surface. Right before 
electroplating with $\mathrm{Cu}$, each wafer was cleaned one last time with 5\% $\mathrm{HF}$ to remove the native oxide from the surface of $\mathrm{Si}$ and produce an electrically conductive surface. The wafers were plated with $\mathrm{Cu}$ in a bath containing an aqueous solution of $0.8 \mathrm{M} \mathrm{CuSO}_{4}$ (and $1 \mathrm{ml}$ ethanol) using a Pt-wire as anode. Plating was performed with a current density of $0.20 \mathrm{~mA} \mathrm{~cm}^{-1}$ for $15 \mathrm{~min}$ to produce a film of $\mathrm{Cu}$ approximately $1 \mu \mathrm{m}$. Finally, the wafers were diced into $5 \times 5 \mathrm{~mm}^{2}$ pieces using a fiber laser, (Speedy 100 fiber 20W, Trotec $®$ ) to produce $\mathrm{Cu}-\mathrm{pSi}$ contacts. All chemicals, unless otherwise stated, were purchased from Sigma.

Attachment of contacts. $\mathrm{Cu}$-pSi: CB-PDMS layers (printed on the PDMS carrier substrate) were protected using a $0.1 \mathrm{~mm}$-thick stencil with $5 \times 5 \mathrm{~mm}^{2}$ patterns, exposing only the bonding sites. The non-plated side of the $\mathrm{Cu}$-pSi contacts and exposed regions of the CB-PDMS layer were treated with $\mathrm{O}_{2}$ plasma ( 0.4 mbar) for 35 s using a Gala Instruments Plasma Prep 5 Cleaner to prime each element for bonding. The components were bonded together by simply bringing them in contact for $30 \mathrm{~s}$. Tin solder ( $\mathrm{Sn63-Pb37)}$ was used to solder $\mathrm{Cu}$ wires on the Cu-plated side of the $\mathrm{Cu}$-pSi contacts at $300{ }^{\circ} \mathrm{C}$ for electrical and mechanical characterization. AgEpoxy: A two-component AgEpoxy mixture (8331S Silver epoxy adhesive, MG Chemicals) is transferred onto the CB-PDMS layers through the same stencil as above using stencil printing. AgEpoxy was dried at $100{ }^{\circ} \mathrm{C}$ for at least $2 \mathrm{~h}$ to minimize malleability and ensure complete curing. Microstructural characterization: SEM micrographs were acquired using a Zeiss Gemini Sigma 300 FEG SEM at $5 \mathrm{keV}$ electron beam energy. For elemental analysis we used an Oxford Instruments X-act X-ray detector. Optical micrographs were obtained with a Brunel SP202XM metallurgical microscope. A Nikon D3200 type camera was attached to the microscope and the images were processed through to a PC in real-time using open-source DigiCamControl software to extract scale parameters. 
Electrical and mechanical characterization. We used a custom-built circuit (Figure S10) to study the piezoresistive behavior of the devices built during electro-mechanical testing. This circuit implements a constant current source with optimal temperature stability that renders wearable electronics, such as the breathing harness or Squeeze-ball that we manufactured, more reliable outdoors or after prolonged use. The circuit can operate using low voltages and can also be powered by the $3.3 \mathrm{~V}$ supply of an Arduino board, which makes it versatile for multiple devices and use-cases. The emitter resistor is selected to control the current through the sensing element and resistance is deduced by measuring the voltage with the known current. We used a second PNP BJT transistor to compensate for voltage drops and temperature fluctuations. The IV curves for the characterization of the metal-semiconductor interfaces were acquired using a Uni-Trend Technology Co., Ltd UT171B multimeter and Tenma 72-2535 type variable benchtop DC power supply. Mechanical tests were performed using an Instron 5543 tensile tester (1 $\mathrm{kN}$ load cell, Bluehill 3 software). All error bars report standard deviation based on the number of samples reported in the caption.

\section{ASSOCIATED CONTENT}

Brief description of Supporting Information. Figure S1: Picture of a batch of stencil-printed CB-PDMS sensing elements. Figure S2: Resistance vs. CB concentration for the CB-PDMS conductive polymer composite. Figure S3: Electron micrographs and elemental analysis of the cross section of Cu-pSi and $\mathrm{Cu}$ electroplated on unetched Si. Figure S4: Electron micrographs of the MACE and $\mathrm{Cu}$ electroplating processes, tensile testing results, and photographs of soldered samples for Si samples with increasing pore sizes and flat Si. Figure S5: Schematic of the condensation plasma bonding reaction. Table S1: Stress and strain values for plasma-treated and pristine samples of PDMS. Figure S6: Detailed strain - stress characteristics for samples of CB- 
PDMS ( 3 mm thickness, 12\% CB content) with AgEpoxy and Cu-pSi contacts. Figure S7:

Maximum stress experiments for pristine and plasma-treated PDMS samples with 3-, 6- and 9mm thickness. Figure S8: Picture of CB-PDMS sensors with Cu-pSi and AgEpoxy contacts. Figure S9: Detailed analysis max. strain at failure under cycling stretching. Figure S10: Schematic of the custom-built constant current circuit for measuring the resistance of the silicone-based piezoresistive CPC sensors. Video V1: Cracking of AgEpoxy during cyclic stretching on the tensile machine (.mp4). Video V2: Demonstration of the wearable breathing monitor with the CB-PDMS sensor and Cu-pSi contacts (.mp4). Video V3: Demonstration of the leg band with the CB-PDMS sensor and Cu-pSi contacts (.mp4). Video V4: Demonstration of the rehabilitation ball (i.e. Squeeze-ball) with the embedded CB-PDMS sensor and Cu-pSi contacts (.mp4).

\section{Acknowledgements}

Firat Güder and Estefania Nunez-Bajo would like to thank The UK Engineering and Physical Sciences Research Council (EPSRC) (EP/R010242/1), General Electric Healthcare and Imperial College, Department of Bioengineering for their generous support. Michael Kasimatis acknowledges EPSRC DTP (Reference: 1846144). Yasin Cotur thanks the Turkish Ministry of Education. Firat Güder, Ji-Seon Kim and Max Grell thank Imperial College Centre for Plastic Electronics and acknowledge EPSRC for Plastic Electronics Doctoral Training Centre (EP/G037515/1 and EP/L016702/1). Firat Güder also acknowledges Agri Futures Lab.

\section{References}


(1) Xu, S.; Zhang, Y.; Cho, J.; Lee, J.; Huang, X.; Jia, L.; Fan, J. a; Su, Y.; Su, J.; Zhang, H.; et al. Stretchable Batteries with Self-Similar Serpentine Interconnects and Integrated Wireless Recharging Systems. Nat. Commun. 2013, 4, 1543.

(2) Lipomi, D. J.; Tee, B. C. K.; Vosgueritchian, M.; Bao, Z. Stretchable Organic Solar Cells. Adv. Mater. 2011, 23 (15), 1771-1775.

(3) Lee, M.; Chen, C.-Y.; Wang, S.; Cha, S. N.; Park, Y. J.; Kim, J. M.; Chou, L.-J.; Wang, Z. L. A Hybrid Piezoelectric Structure for Wearable Nanogenerators. Adv. Mater. 2012, 24 (13), 1759-1764.

(4) Ranunkel, O.; Güder, F.; Arora, H. Soft Robotic Surrogate Lung. ACS Appl. Bio Mater. 2019, 2 (4), 1490-1497.

(5) Koh, A.; Kang, D.; Xue, Y.; Lee, S.; Pielak, R. M.; Kim, J.; Hwang, T.; Min, S.; Banks, A.; Bastien, P.; et al. A Soft, Wearable Microfluidic Device for the Capture, Storage, and Colorimetric Sensing of Sweat. Sci. Transl. Med. 2016, 8 (366), 366ra165.

(6) Park, J.; Kim, J.; Kim, K.; Kim, S. Y.; Cheong, W. H.; Park, K.; Song, J. H.; Namgoong, G.; Kim, J. J.; Heo, J.; et al. Wearable, Wireless Gas Sensors Using Highly Stretchable and Transparent Structures of Nanowires and Graphene. Nanoscale 2016, 8 (20), 10591-10597.

(7) Bowden, N.; Brittain, S.; Evans, A. G.; Hutchinson, J. W.; Whitesides, G. M. Spontaneous Formation of Ordered Structures in Thin Films of Metals Supported on an Elastomeric Polymer. Nature 1998, 393 (6681), 146-149.

(8) Güder, F.; Ainla, A.; Redston, J.; Mosadegh, B.; Glavan, A.; Martin, T. J.; Whitesides, G. M. Paper-Based Electrical Respiration Sensor. Angew. Chemie - Int. Ed. 2016, 55 (19), 
$5727-5732$.

(9) Dincer, C.; Bruch, R.; Costa-Rama, E.; Fernández-Abedul, M. T.; Merkoçi, A.; Manz, A.; Urban, G. A.; Güder, F. Disposable Sensors in Diagnostics, Food, and Environmental Monitoring. Adv. Mater. 2019, 31 (30), 1806739.

(10) Imani, S.; Bandodkar, A. J.; Mohan, A. M. V.; Kumar, R.; Yu, S.; Wang, J.; Mercier, P. P. A Wearable Chemical-Electrophysiological Hybrid Biosensing System for Real-Time Health and Fitness Monitoring. Nat. Commun. 2016, 7 (May), 11650.

(11) Giorgino, T.; Tormene, P.; Lorussi, F.; De Rossi, D.; Quaglini, S. Sensor Evaluation for Wearable Strain Gauges in Neurological Rehabilitation. IEEE Trans. Neural Syst. Rehabil. Eng. 2009, 17 (4), 409-415.

(12) Carpi, F.; DeRossi, D. Electroactive Polymer-Based Devices for e-Textiles in Biomedicine. IEEE Trans. Inf. Technol. Biomed. 2005, 9 (3), 295-318.

(13) Duan, S.; Yang, K.; Wang, Z.; Chen, M.; Zhang, L.; Zhang, H.; Li, C. Fabrication of Highly Stretchable Conductors Based on 3D Printed Porous Poly(Dimethylsiloxane) and Conductive Carbon Nanotubes/Graphene Network. ACS Appl. Mater. Interfaces 2016, 8 (3), 2187-2192.

(14) Bonyár, A.; Sántha, H.; Ring, B.; Varga, M.; Gábor Kovács, J.; Harsányi, G. 3D Rapid Prototyping Technology (RPT) as a Powerful Tool in Microfluidic Development. Procedia Eng. 2010, 5, 291-294.

(15) Lu, T.; Finkenauer, L.; Wissman, J.; Majidi, C. Rapid Prototyping for Soft-Matter Electronics. Adv. Funct. Mater. 2014, 24 (22), 3351-3356. 
(16) Bartlett, M. D.; Markvicka, E. J.; Majidi, C. Rapid Fabrication of Soft, Multilayered Electronics for Wearable Biomonitoring. Adv. Funct. Mater. 2016, 8496-8504.

(17) Tavakoli, M.; Rocha, R.; Osorio, L.; Almeida, M.; de Almeida, A.; Ramachandran, V.; Tabatabai, A.; Lu, T.; Majidi, C. Carbon Doped PDMS: Conductance Stability over Time and Implications for Additive Manufacturing of Stretchable Electronics. J. Micromechanics Microengineering 2017, 27 (3), 035010.

(18) Amjadi, M.; Kyung, K. U.; Park, I.; Sitti, M. Stretchable, Skin-Mountable, and Wearable Strain Sensors and Their Potential Applications: A Review. Adv. Funct. Mater. 2016, 26 (11), 1678-1698.

(19) Amjadi, M.; Pichitpajongkit, A.; Lee, S.; Ryu, S.; Park, I. Highly Stretchable and Sensitive Strain Sensor Based on Silver-Elastomer Nanocomposite. ACS Nano 2014, 8 (5), 51545163.

(20) Roh, E.; Hwang, B.-U.; Kim, D.; Kim, B.-Y.; Lee, N.-E. Stretchable, Transparent, Ultrasensitive, and Patchable Strain Sensor for Human-Machine Interfaces Comprising a Nanohybrid of Carbon Nanotubes and Conductive Elastomers. ACS Nano 2015, 9 (6), $6252-6261$.

(21) Amjadi, M.; Yoon, Y. J.; Park, I. Ultra-Stretchable and Skin-Mountable Strain Sensors Using Carbon Nanotubes-Ecoflex Nanocomposites. Nanotechnology 2015, 26 (37), 375501.

(22) Sun, Y.; Choi, W. M.; Jiang, H.; Huang, Y. Y.; Rogers, J. A. Controlled Buckling of Semiconductor Nanoribbons for Stretchable Electronics. Nat. Nanotechnol. 2006, 1 (3), 
201-207.

(23) Jeong, S.; Hjort, K.; Wu, Z.; Jeong, S. H.; Hjort, K.; Wu, Z. Tape Transfer Printing of a Liquid Metal Alloy for Stretchable RF Electronics. Sensors 2014, 14 (9), 16311-16321.

(24) van den Brand, J.; Kusters, R.; Barink, M.; Dietzel, A. Flexible Embedded Circuitry: A Novel Process for High Density, Cost Effective Electronics. Microelectron. Eng. 2010, 87 (10), 1861-1867.

(25) Ruh, D.; Reith, P.; Sherman, S.; Theodor, M.; Ruhhammer, J.; Seifert, A.; Zappe, H. Stretchable Optoelectronic Circuits Embedded in a Polymer Network. Adv. Mater. 2014, 26 (11), 1706-1710.

(26) Salowitz, N.; Guo, Z.; Li, Y.-H.; Kim, K.; Lanzara, G.; Chang, F.-K. Bio-Inspired Stretchable Network-Based Intelligent Composites. J. Compos. Mater. 2013, 47 (1), 97105.

(27) Mach, P.; Geczy, A.; Polanský, R.; Bušek, D. Glass Transition Temperature of Nanoparticle-Enhanced and Environmentally Stressed Conductive Adhesive Materials for Electronics Assembly. J. Mater. Sci. Mater. Electron. 2019, 30 (5), 4895-4907.

(28) Owen, M. J. Surface Tension of Polytrifluoropropylmethylsiloxane. J. Appl. Polym. Sci. 1988, 35 (4), 895-901.

(29) Kim, J.; Banks, A.; Cheng, H.; Xie, Z.; Xu, S.; Jang, K. I.; Lee, J. W.; Liu, Z.; Gutruf, P.; Huang, X.; et al. Epidermal Electronics with Advanced Capabilities in Near-Field Communication. Small 2015, 11 (8), 906-912.

(30) Xu, S.; Zhang, Y.; Jia, L.; Mathewson, K. E.; Jang, K.-I.; Kim, J.; Fu, H.; Huang, X.; Chava, 
P.; Wang, R.; et al. Soft Microfluidic Assemblies of Sensors, Circuits, and Radios for the Skin. Science 2014, 344 (6179), 70-74.

(31) Jang, K.-I.; Banks, A.; Won, P.; Li, Y.; Rogers, J. A.; Kim, B. H.; Xu, R.; Ma, S.; Lee, J. W.; Paik, U.; et al. Soft, Thin Skin-Mounted Power Management Systems and Their Use in Wireless Thermography. Proc. Natl. Acad. Sci. 2016, 113 (22), 6131-6136.

(32) Biswas, S.; Reiprich, J.; Cohrs, T.; Arboleda, D. T.; Schoeberl, A.; Mozafari, M.; Schlag, L.; Stauden, T.; Pezoldt, J.; Jacobs, H. O. 3D Metamorphic Stretchable Microphone Arrays. Adv. Mater. Technol. 2017, 2 (10), 1700131.

(33) Kaneko, H.; Horie, J. Breathing Movements of the Chest and Abdominal Wall in Healthy Subjects. Respir. Care 2012, 57 (9), 1442-1451.

(34) Lu, N.; Lu, C.; Yang, S.; Rogers, J. Highly Sensitive Skin-Mountable Strain Gauges Based Entirely on Elastomers. Adv. Funct. Mater. 2012, 22 (19), 4044-4050.

(35) Kang, I.; Schulz, M. J.; Kim, J. H.; Shanov, V.; Shi, D. A Carbon Nanotube Strain Sensor for Structural Health Monitoring. Smart Mater. Struct. 2006, 15 (3), 737-748.

(36) Güder, F.; Yang, Y.; Kücìukbayrak, U. M.; Zacharias, M. Tracing the Migration History of Metal Catalysts in Metal-Assisted Chemically Etched Silicon. ACS Nano 2013, 7 (2), 15831590.

(37) Grell, M.; Dincer, C.; Le, T.; Lauri, A.; Nunez Bajo, E.; Kasimatis, M.; Barandun, G.; Maier, S. A.; Cass, A. E. G.; Güder, F. Autocatalytic Metallization of Fabrics Using Si Ink, for Biosensors, Batteries and Energy Harvesting. Adv. Funct. Mater. 2019, 29 (1), 1804798.

(38) Fukami, K.; Kobayashi, K.; Matsumoto, T.; Kawamura, Y. L.; Sakka, T.; Ogata, Y. H. 
Electrodeposition of Noble Metals into Ordered Macropores in P-Type Silicon. J. Electrochem. Soc. 2008, 155 (6), D443.

(39) Butrón-García, M. I.; Jofre-Reche, J. A.; Martín-Martínez, J. M. Use of Statistical Design of Experiments in the Optimization of Ar-O2 Low-Pressure Plasma Treatment Conditions of Polydimethylsiloxane (PDMS) for Increasing Polarity and Adhesion, and Inhibiting Hydrophobic Recovery. Appl. Surf. Sci. 2015, 332, 1-11.

(40) Liston, E. M.; Martinu, L.; Wertheimer, M. R. Plasma Surface Modification of Polymers for Improved Adhesion: A Critical Review. J. Adhes. Sci. Technol. 1993, 7 (10), 10911127.

(41) So, J. H.; Tayi, A. S.; Güder, F.; Whitesides, G. M. Stepped Moduli in Layered Composites. Adv. Funct. Mater. 2014, 24 (45), 7197-7204.

(42) Roy, S.; Kumar, A. Effect of Particle Size on Mixed-Mode Fracture of Nanographene Reinforced Epoxy and Mode I Delamination of Its Carbon Fiber Composite. Compos. Struct. 2017, 181, 1-8.

(43) Kashfipour, M. A.; Mehra, N.; Zhu, J. A Review on the Role of Interface in Mechanical, Thermal, and Electrical Properties of Polymer Composites. Adv. Compos. Hybrid Mater. 2018, 1 (3), 415-439.

(44) Seyedin, S.; Razal, J. M.; Innis, P. C.; Jalili, R.; Wallace, G. G. Compositional Effects of Large Graphene Oxide Sheets on the Spinnability and Properties of Polyurethane Composite Fibers. Adv. Mater. Interfaces 2016, 3 (5), 1500672.

(45) Diani, J.; Fayolle, B.; Gilormini, P. A Review on the Mullins Effect. Eur. Polym. J. 2009, 
$45(3), 601-612$.

(46) Mazurek, P.; Vudayagiri, S.; Skov, A. L. How to Tailor Flexible Silicone Elastomers with Mechanical Integrity: A Tutorial Review. Chem. Soc. Rev. 2019, 48, 1448.

(47) Ma, W.; Zhang, W.; Li, P.; Hu, J.; Li, G.; Sang, S. I-V Characteristics of the MetalSemiconductor Junction. In Proceedings - 2012 International Conference on Computing, Measurement, Control and Sensor Network, CMCSN 2012; IEEE, 2012; 195-198.

(48) Elhadidy, H.; Sikula, J.; Franc, J. Symmetrical Current-Voltage Characteristic of a MetalSemiconductor-Metal Structure of Schottky Contacts and Parameter Retrieval of a CdTe Structure. Semicond. Sci. Technol. 2012, 27 (1), 015006.

(49)

Halas, S.; Durakiewicz, T. Work Functions of Elements Expressed in Terms of the Fermi Energy and the Density of Free Electrons. J. Phys. Condens. Matter 1998, 10 (48), 1081510826.

(50) US Department of Health and Human Services, https://householdproducts.nlm.nih.gov/cgi -bin/household/brands?id=161\&tbl=chem , (Accessed April 2019).

(51) Kadilak, A. L.; Liu, Y.; Shrestha, S.; Bernard, J. R.; Mustain, W. E.; Shor, L. M. Selective Deposition of Chemically-Bonded Gold Electrodes onto PDMS Microchannel Side Walls. J. Electroanal. Chem. 2014, 727, 141-147.

(52) Kong, J.-H.; Jang, N.-S.; Kim, S.-H.; Kim, J.-M. Simple and Rapid Micropatterning of Conductive Carbon Composites and Its Application to Elastic Strain Sensors. Carbon N. Y. 2014, 77, 199-207.

(53) Zheng, Y.; Li, Y.; Li, Z.; Wang, Y.; Dai, K.; Zheng, G.; Liu, C.; Shen, C. The Effect of 
Filler Dimensionality on the Electromechanical Performance of Polydimethylsiloxane Based Conductive Nanocomposites for Flexible Strain Sensors. Compos. Sci. Technol. 2017, 139, 64-73.

(54) Lai, R. A.; Hymel, T. M.; Narasimhan, V. K.; Cui, Y. Schottky Barrier Catalysis Mechanism in Metal-Assisted Chemical Etching of Silicon. ACS Appl. Mater. Interfaces 2016, 8 (14), 8875-8879.

(55) Boehler, C.; Güder, F.; Kücükbayrak, U. M.; Zacharias, M.; Asplund, M. A Simple Approach for Molecular Controlled Release Based on Atomic Layer Deposition Hybridized Organic-Inorganic Layers. Sci. Rep. 2016, 6 (1), 19574. 
Monolithically integrated nanoporous Si-based electrical contacts form covalent bonds with silicone-based conductive composites used for sensing and physiological monitoring applications. Covalent interfacial bonding and a move away from brittle conductive adhesives increases the mechanical strength and improves the reliability of the electrical signal for electrical contacts used in stretchable electronics with scalable manufacturing for wearable devices.

Michael Kasimatis*, Estefania Nunez-Bajo, Max Grell, Yasin Cotur, Giandrin Barandun, Ji-Seon

Kim, Firat Güder*

Monolithic Solder-on Nanoporous Si-Cu Contacts for Stretchable Silicone Composite Sensors
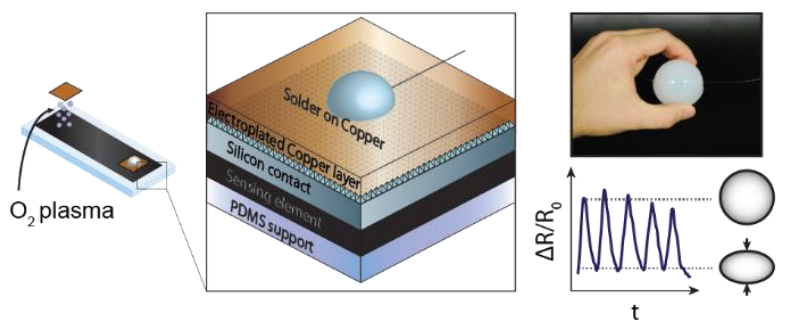